\title{
Smoking Attenuates the Beneficial Effect of Physical Activity on Intima-Media Thickness of Carotid Artery in Young and Middle-Aged Smokers
}

\author{
Hiroyuki Katano a, b, d, Kazuo Yamada ${ }^{\mathrm{b}}$, Masahiro Ohno ${ }^{\mathrm{c}}$
}

\begin{abstract}
Background: Though the favorable effect of physical activity and the hazardous effect of smoking on the progression of atherosclerosis have been well studied, the interactive effect of the two factors has seldom been investigated.
\end{abstract}

Methods: A total of 1,090 subjects from among examinees of screening for brain diseases (an annual medical checkup of the brain) from April 2007 to March 2008 were studied to clarify the effect of physical activity on current, former and never smokers in relation to maximum carotid intima-media thickness (IMT). Univariate and multivariate analyses were performed to investigate the relationship between maximum IMT and independent variables such as age, gender, coexisting disease, physical activity, alcohol drinking, family history, symptoms, BMI, systolic blood pressure, diastolic blood pressure, blood sugar, total cholesterol, HDL cholesterol, triglyceride, Bathel index and time since smoking cessation.

Results: Univariate analysis revealed that only never smokers exhibited a significant relationship between physical activity and maximum IMT. Multiple regression analyses performed for three age specific groups $(\leq 49,50-59, \geq 60)$, demonstrated that physical activity was not a significant predictor of maximum IMT in current

Manuscript accepted for publication December 2, 2011

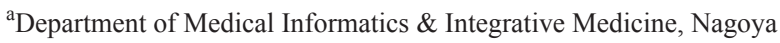
City University Graduate School of Medical Sciences, Nagoya, Japan ${ }^{b}$ Department of Neurosurgery, Nagoya City University Graduate School of Medical Sciences, Nagoya, Japan

${ }^{c}$ Health Management Center, Chunichi Hospital, Nagoya, Japan

${ }^{\mathrm{d}}$ Corresponding author: Hiroyuki Katano, Department of Medical Informatics \& Integrative Medicine, Nagoya City University Graduate School of Medical Sciences 1, Kawasumi, Mizuho-cho, Mizuho-ku, Nagoya 467-8601, Japan. Email: katano@med.nagoya-cu.ac.jp

doi:10.4021/jnr74w and former smokers 49 years old and younger, and current smokers 50 - 59 years old, though the other multivariate analyses showed no meaningful results, including for never smokers.

Conclusions: The present study revealed that for young and middle-aged current smokers, the favorable effect of physical activity on the prevention of carotid atherosclerosis might be attenuated by detrimental effect of smoking.

Keywords: Physical activity; Smoking; Carotid artery; Intimamedia thickness; Atherosclerosis

\section{Introduction}

A great deal of evidence has shown that appropriate physical exercise may promote health. It has been reported that an adequate amount of physical activity reduces cardiovascular and cerebrovascular events, and an increased risk of stroke is associated with physical inactivity [1]. Concerning physical activity and intima-media thickness (IMT), most reports demonstrate the association of physical inactivity and increase in IMT, though some contradictory reports exist [2-4].

Cigarette smoking is also known to increase the risk of cerebrovascular as well as cardiovascular events. Cigarette smoking has been well-documented as a risk factor for ischemic stroke in general, with relative risks of 1.5 - 5.7 [5]. It is also well-reported that smoking degrades carotid IMT [6], a well-known surrogate marker for atherosclerosis [7].

However, with the exception of portions in studies of separate physical activities and smoking effects $[1,8,9]$, few investigators have analyzed in detail the interactive relationship of physical activity and smoking to atherosclerosis. We investigated examinees of the "brain dock", part of an annual physical examination ("ningen dock" or "human dry dock") that screens for cerebral diseases, and analyzed results to clarify the effect of physical activity on smokers with regard to atherosclerotic changes of the carotid artery. We employed maximum IMT of the carotid artery to evaluate carotid atherosclerosis and focused on whether smoking attenuates the favorable effect of physical activity on carotid 
atherosclerosis.

\section{Methods}

\section{Subjects}

A total of 2012 subjects (1369 men, 643 women, aged 54.25 \pm 9.58 years) visited the Health Management Center at Chunichi Hospital (Nagoya, Japan) from April 2007 to March 2008 for screening of brain diseases (medical checkup of the brain) as part of an annual physical examination. A self-administered questionnaire including smoking status, physical activity, coexisting disease, alcohol use, family history and present symptoms was applied. The set of the brain-checkup examination included carotid ultrasonography, blood pressure measurement and routine blood tests.

A clerk randomly collected 20 records once or twice a week without consideration of the identity of the subjects. Fifty-eight collections in the period above resulted in a randomly selected total of 1,160 subjects. Among them, 30 subjects had no data for blood sampling due to the subjects' opting out. Twenty-eight subjects ceased smoking within 3 years and 7 persons wrote 0 for the period they had quit smoking, with no further description of smoking history. One subject had no description of smoking history and 4 had other incomplete items on the questionnaire. Finally, 1,090 subjects were enrolled the present study. The study design was approved by the local ethical committee and the ethics guidelines for clinical studies by the Japanese Health Labor and Welfare Ministry (2007) were strictly observed.

\section{Carotid Ultrasonography}

Carotid IMT of the common and internal carotid arteries were evaluated using high-resolution sonography equipped with a linear transducer at $7.5 \mathrm{MHz}$ in B mode (HDI5000 SonoCT, HITACHI, Japan) by 3 trained investigators with no knowledge of the background of the subjects. IMT of each common carotid artery (CCA) and internal carotid artery (ICA) was measured on a frozen frame in the longitudinal plane. The maximum IMT was defined as the thickest wall of the lateral and medial walls of the CCAs and ICAs on both sides. Plaques are included when maximum IMT is measured.

\section{Items in analysis and definitions}

Smoking status was classified into 3 categories: (1) Current smoker: a person who has smoked at least one cigarette per day for the preceding three years or more. The Bathel index was calculated as the number of cigarettes smoked per day multiplied by of years of smoking. (2) Former smoker: a person who has smoked at least one cigarette per day at some period, but has not smoked for the preceding 3 years or more. (3) Never smoker: a person who has never smoked in his or her lifetime.

Self-reported physical activity was classified into 3 categories: (1) Low physical activity: an inactive person who does not meet the criteria for moderate or high physical activities. (2) Moderate physical activity: daily walking for at least one hour or comparable activity. (3) High physical activity: weekly exercising or sports (jogging, swimming, training at gym, tennis, golf, etc.) for at least half an hour twice a week. They were scored 0,1 , and 2 , respectively.

Alcohol use was categorized as 0,1 , or 2 , which corresponded to none or less than 1 unit/day (1 unit equal to 20 $\mathrm{g}$ of pure alcohol), $1-3$ units/day and more than 3 units/day, respectively.

Numbers of coexisting diseases were categorized as 0 , $1,2,3$ or more. Diseases were counted if they were known to be risk factors for atherosclerosis, for instance hypertension, diabetes mellitus, hyperlipidemia, cerebral infarction and ischemic heart disease.

Family history was counted if the examinee's parents and siblings had diseases known to be risk factors for atherosclerosis, such as hypertension, diabetes mellitus, hyperlipidemia, cerebral infarction and ischemic heart disease. They were counted and categorized as $0,1,2,3$ and more.

Body mass index (BMI) was calculated as the weight in kilograms divided by the square of the height in meters.

The number of present symptoms which examinees described was counted and categorized as 0, 1, 2, 3 and more, and included such symptoms as headache, numbness of limbs, tinnitus, floating feelings, dizziness, memory loss and speaking problems.

Among routine blood tests, blood sugar, total cholesterol, high-density-lipoprotein (HDL) -cholesterol and triglyceride were chosen for analysis. As low-density-lipoprotein (LDL) -cholesterol was optionally included in testing by examinees' choice and more than a few of them had no values recorded, the item was excluded from the present analysis.

\section{Statistical analysis}

All statistical evaluations were performed with statistical software (JMP version 5, SPS, NC; Statview version 4.5, $\mathrm{SPS}, \mathrm{NC}$ ) and all results are presented as mean $\pm \mathrm{SD}$. To test whether a difference existed between more than two groups, Kruskal Wallis and Chi-square tests were used. A multiple test procedure (Bonferroni correction) was employed. P values less than 0.05 were considered to indicate a significant difference. A univariate selection of candidate predictor variables for maximum IMT was conducted. The correlation between two continuous variables was quantified by using Spearman rank order correlation coefficients ( $\rho$ values) with a zero correlation except for categoric variable (sex) using the Mann-Whitney U test. We applied maximum IMT as a 


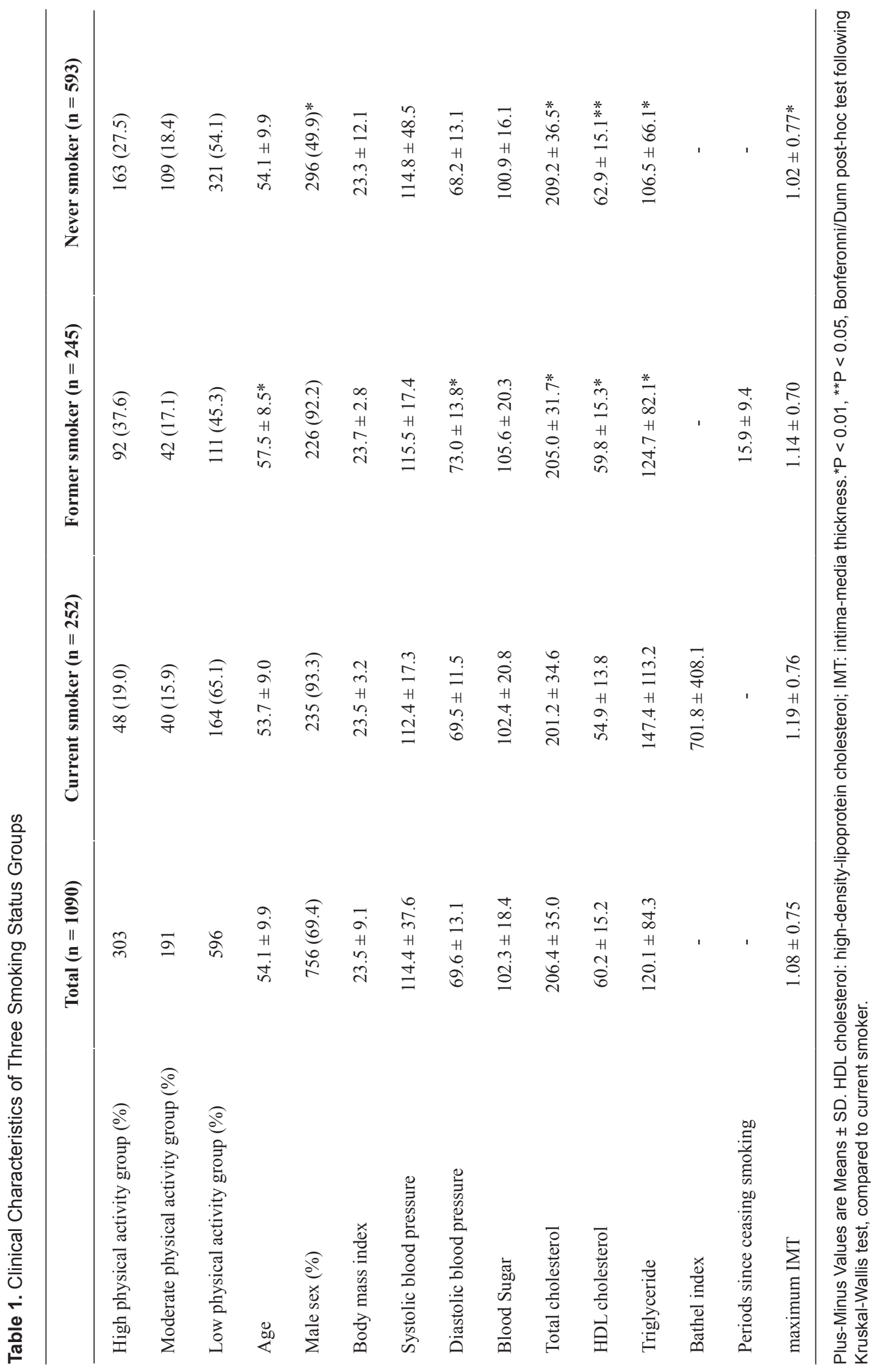


Table 2. Multigroup Analysis of Maximum IMT With Reference to Smoking Status and Each Intensity of Physical Activity

\section{Current-smoker groups $\quad$ Former-smoker groups $\quad$ Never-smoker groups}

\begin{tabular}{|c|c|c|c|}
\hline $\mathrm{P}^{*}$ & 0.657 & 0.252 & 0.003 \\
\hline $\mathrm{P}^{* *}$ (low vs moderate physical activity groups) & - & - & 0.343 \\
\hline $\mathrm{P}^{* *}$ (moderate vs high physical activity groups) & - & - & 0.253 \\
\hline $\mathrm{P}^{* *}$ (low vs high physical activity groups) & - & - & 0.011 \\
\hline
\end{tabular}

$\mathrm{P}^{*}$ : Kruskal-Wallis test, $\mathrm{P}^{* *}$ : Bonferroni/Dunn post-hoc test.

dependent variable and independent variables as candidate predictors were as follows: age, sex, coexisting disease, physical activity, alcohol use, family history, symptoms, body mass index (BMI), systolic blood pressure, diastolic blood pressure, blood sugar, total cholesterol, HDL-cholesterol and triglycerides. All subjects were subsequently divided into three age groups ( $\leq 49,50-59,60 \leq$ years old) and multivariate analysis was performed for each age group. Before the multiple linear regression analysis was performed, nine dependent variables were selected by cluster analysis (the Ward method was applied to calculate the distance of variables) to avoid multicolinearity among the dependent variables. Sex was included in the model as a variable coded as 1 (for male) or 0 (for female). Multivariate regression analysis with the least squares method for the nine selected dependent valuables was performed for each age group with a status of three for smoking. Dependent valuables with $\mathrm{p}$ values $<0.05$ for the $\mathrm{F}$ statistics in the analysis with $\mathrm{R}$ squared $\geq 0.2$ were considered as significant predictors for the independent variable.

\section{Results}

The enrolled 1,090 examinees consisted of 756 males and 334 females. The clinical characteristics of subjects and mean values of continuous variables for each group of smoking status are summarized in Table 1. Average ages of current smokers were rather lower than those of former smokers and current smokers had a lower proportion of females than never smokers $(\mathrm{P}<0.01)$. Mean values for total and HDLcholesterol of current smokers were lower than other groups, while their triglyceride values were higher. Mean values for maximum IMT were higher in current smokers than in never smokers $(\mathrm{P}<0.01)$.

The difference between the three physical activity groups concerning maximum IMT in each smoking status group was analyzed (Table 2). In current and former smokers, no significant differences were found among the three physical activity groups, whereas in never smokers, a significant difference was found concerning maximum IMT between low and high physical activity groups $(\mathrm{P}=0.011)$.

Univariate analysis of the independent variables also showed a significant reverse association between physical activity and maximum IMT only in the never smoker group $(\rho=-0.136, P=0.001$, Table 3$)$.

Following the division of the three age subgroups $(\leq 49$, 50 - 59, $60 \leq$ years old) (Table 4), multivariate analysis was tested for people of every smoking status in each age group regarding maximum IMT in relation to the selected nine independent variables by cluster analysis (Table 4). In the group with subjects 49 years old and younger, physical activity was not selected as a significant predictor for maximum IMT in current and former smokers $(\mathrm{F}=1.024, \mathrm{P}=0.315$ and $\mathrm{F}=0.968, \mathrm{P}=0.334$, respectively). In current smokers, physical activity was not chosen as a significant predictor for maximum IMT in the 50 - 59-year-old group $(\mathrm{F}=0.125, \mathrm{P}=$ 0.724). In groups for other ages and for smoking status, the analyses were not chosen for low R-square values.

\section{Discussion}

Physical activity seems a plausible factor in decreasing cardiovascular and cerebrovascular event risks. Sacco et al. [1] found through analysis of the Northern Manhattan Stroke Study, a population-based incidence and case-control study, that leisure-time physical activity was significantly protective for stroke and that a dose-response relationship was shown for both intensity and duration of physical activity. $\mathrm{Hu}$ et al. [10] prospectively followed 47,721 subjects and found that a high level of leisure time physical activity reduced the risk of 
Table 3. Univariate Analysis for Maximum IMT With Each Factor in Three Smoking Status Groups

\begin{tabular}{|c|c|c|c|c|c|c|}
\hline \multirow[b]{2}{*}{ Variables } & \multicolumn{2}{|c|}{ Current smoker } & \multicolumn{2}{|c|}{ Former smoker } & \multicolumn{2}{|c|}{ Never smoker } \\
\hline & $\rho$ & $\mathrm{P}$ & $\rho$ & $\mathrm{P}$ & $\rho$ & $\mathrm{P}$ \\
\hline Physical activity & -0.053 & 0.403 & -0.025 & 0.695 & $-0.136^{*}$ & 0.001 \\
\hline Age & 0.435 & $<0.0001$ & 0.313 & $<0.0001$ & 0.474 & $<0.0001$ \\
\hline Coexisting disease & 0.284 & $<0.0001$ & 0.215 & 0.001 & 0.272 & $<0.0001$ \\
\hline Alcohol drinking & 0.050 & 0.389 & 0.033 & 0.602 & -0.020 & 0.634 \\
\hline Family history & -0.007 & 0.916 & 0.066 & 0.171 & -0.016 & 0.689 \\
\hline Present symptom & 0.013 & 0.835 & 0.088 & 0.299 & -0.060 & 0.144 \\
\hline Body mass index & 0.022 & 0.728 & 0.048 & 0.451 & 0.108 & 0.009 \\
\hline Systolic blood pressure & 0.146 & 0.021 & 0.299 & $<0.0001$ & 0.255 & $<0.0001$ \\
\hline Diastolic blood pressure & -0.061 & 0.334 & 0.212 & 0.001 & 0.179 & $<0.0001$ \\
\hline Blood Sugar & 0.065 & 0.302 & 0.127 & 0.047 & 0.192 & $<0.0001$ \\
\hline Total cholesterol & 0.108 & 0.089 & 0.034 & 0.592 & 0.181 & 0.013 \\
\hline HDL cholesterol & -0.033 & 0.598 & -0.057 & 0.376 & -0.127 & 0.002 \\
\hline Triglyceride & -0.109 & 0.083 & 0.040 & 0.531 & 0.181 & $<0.0001$ \\
\hline Bathel index & 0.365 & $<0.0001$ & - & - & - & - \\
\hline Periods since ceasing smoking & - & - & -0.161 & 0.012 & - & - \\
\hline Male sex $\uparrow$ & - & 0.071 & - & 0.007 & - & 0.001 \\
\hline
\end{tabular}

Spearman correlation test except Mann-Whitney analysis for gender †. IMT: intima-media thickness; HDL cholesterol: high-densitylipoprotein cholesterol.

all subtypes of stroke, and that active daily commuting also reduced the risk of ischemic stroke. Through a meta-analysis of 23 studies (18 cohort and 5 case-control), Lee et al. [11] found that highly active individuals had a $27 \%$ lower risk of stroke incidence or mortality (relative risk 0.73) than lowactive individuals, and that moderately and highly active individuals had lower risk of both ischemic and hemorrhagic strokes than low-active individuals. Wendel-Vos et al. [12] reported from the results of meta-analysis of observational data from 31 publications that moderately intense physical activity, compared with inactivity, showed a protective effect on total stroke for both occupational (relative risk: RR $=0.64)$ and leisure time physical activity $(R R=0.85)$. High level occupational physical activity protected against ischemic stroke compared with both moderate $(\mathrm{RR}=0.77)$ and inactive occupational levels $(\mathrm{RR}=0.57)$.

Gillum et al. [9] investigated 5,852 persons and found low and non-recreational activity in women as well as men were associated with an increased risk of stroke (relative risk $1.83 \%$ ). Similar mechanisms that account for the protective effect of physical activity against coronary heart disease may also be postulated for the protective effect against stroke: reduction of blood pressure, raising HDL cholesterol, and the like. Endres et al. [13] demonstrated that regular physical activity reduced brain injury after acute focal cerebral ischemia in an animal model. They demonstrated that regular physical activity of mice on a treadmill upregulated eNOS expression and augmented reduction of cerebral infarct size and brain swelling, while the effect was completely absent in eNOSdeficient mice.

Cigarette smoking is a well-known risk factor for ischemic stroke, and various studies have reported on it. Love et al. [14] inspected young adults (15 - 45 years old) and reported that a smoker was 1.6 times more likely to have a cerebral infarction than a nonsmoker, and that there was a cumulative dose effect with each additional pack-year, causing a greater 


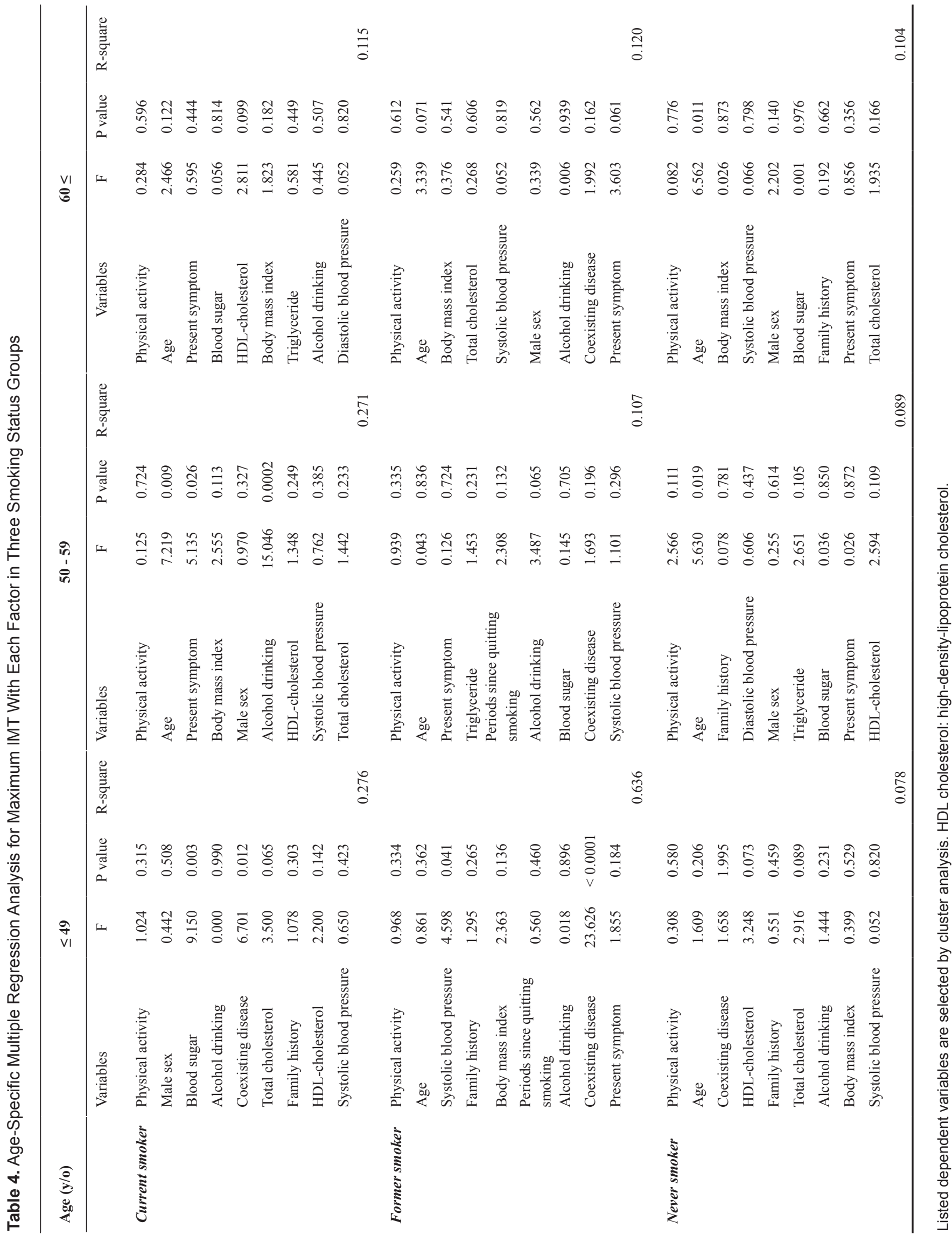


risk of cerebral infarction. Robbins et al. [15] demonstrated that a dose-dependent increased risk of ischemic stroke was also reported in male physicians, with a relative risk of 2.0 when smoking 1-19 cigarettes per day and 2.7 when smoking more than 20 cigarettes per day. Bhat et al. [16] analyzed the data from the Stroke Prevention in Young Women Study, a population-based case-control study of risk factors for ischemic stroke in women aged 15 - 49 years old, and reported a strong dose-response relationship between cigarette smoking and ischemic stroke risk in young women. Mast et al. [17] collected data from two prospective studies and showed that smoking was significantly associated with highgrade carotid artery stenosis $(>60 \%)$, especially in white smokers who smoked 20 pack-years or more, and that this effect was independent of other risk factors. Regarding IMT, Nakashima et al. [18] reported a positive correlation between maximum IMT and smoking in hemodialysis patients, but no correlation between mean IMT and smoking. They considered the possibility that mean IMT was related merely to arterial wall thickening, while maximum IMT was related to atheroma formation. They reported mean IMT correlated with age, hypertension and dyslipidemia, while maximum IMT correlated with age, diabetes and smoking. Adachi et al. [19] also reported that smoking status was not a risk factor for mean IMT, though positive associations between mean IMT and both blood pressure and low HDL cholesterol were found. Bots et al. [20] recommended the use of mean maximum IMT rather than mean common IMT as the primary outcome measure in random controlled trials. Taking into account these various findings, in this study we applied maximum IMT rather than mean IMT to investigate the relationship between smoking, physical activity and atherosclerosis.

The interaction of smoking and physical activity on stroke has been less well studied, except in portions of studies focused on separate physical activities and smoking effects. In most studies, smoking factors were adjusted in the model as one of the undesignated risk factors [8]. In their work concerning leisure-time physical activity and ischemic stroke risk, Sacco et al. [1] stated briefly that the protective effect of physical activity did not differ according to smoking status like other risk factors such as hypertension, diabetes and cardiac disease. Gillum et al. [9] speculated that high recreational physical activity was less protective in white male smokers than in nonsmokers, but did not produce detailed data.

In our study, we initially found from the comparison among three groups of physical activities of each smoking group for maximum IMT that only never smokers demonstrated a significant difference between low and high physical activity groups. Univariate analysis performed to evaluate the contribution of physical activity to carotid maximum IMT in each group of smoking status also revealed that only never smokers showed a significant difference, while current and former smokers did not. These results indicate that never smokers with high physical activity tend to have lower IMT, whereas smokers tend to have higher IMT even with high physical activity. This suggests the possibility that the favorable effect of physical activity might be countered by smoking.

Age-specific multiple linear regression analysis of the items did not select physical activity as a significant predictor of maximum IMT for current smokers in the 49 year-old and younger and 50 - 59 year-old groups. In the 49 year-old and younger group, physical activity was not chosen as a predictor of maximum IMT either for former smokers. These results could be interpreted as the possible attenuation of the beneficial effect of physical activity by the harmful effect of smoking, as was shown in univariate analysis. However, as no other analyses were selected due to low R-square values in the present multivariate analysis including the never smoker group, another interpretation of the above results might be that maximum IMT was affected by multiple complex factors other than the dependent factors selected by cluster analysis, including physical activity, which were well-known factors related to atherosclerosis.

However, at least for young and middle-aged current smokers under 59 years old, beneficial effect of exercise or daily physical activity unfortunately appears to be offset by the hazardous effect of smoking.

We defined a former smoker as a person who has smoked at least one cigarette per day at some period, but has not smoked for the preceding 3 years or more. Stroke risk decreases significantly 3 years after cessation of cigarette smoking and is at the level of nonsmokers by 5 years $[21,22]$. The Framingam study [21] indicated that the risk of stroke decreased significantly 2 years after stopping smoking and was at the level of non-smokers by 5 years after cessation. Within 2 years of quitting smoking, the relative risk of stroke was reduced by $22 \%$ compared with continued smoking [22]. Though the average period since quitting smoking was as long as $15.9 \pm 9.1$ years, physical activity was not chosen as a predictor of maximum IMT in former smokers in the youngest age group ( $\leq 49$ years old) like in current smokers. The fact that the present study did not account for the amount and duration of smoking before cessation for the former smokers may have affected these results.

This study has some limitations. First, though the subjects were randomly selected each week throughout the targeted period by a person unconnected with the study, there remains the possibility of selection bias. Secondly, inter- and intraobserver variability may have been possible in the carotid IMT measurement by ultrasonography, though all three examiners were well-trained experts. Third, physical activities in the study were classified into 3 groups according to the intensity of the activity, but were not quantitatively determined using criteria such as the MET score or IPAQ (International Physical Activity Questionnaire [23, 24] and divided into finer categories. Fourth, medication such as 
statin [25], antihypertensive [26] and antiplatelet drugs [27] which are reported to decrease IMT, though mostly mean IMT, were not considered in this study. Lastly, this study did not take into consideration exposure of non-smokers to environmental smoke, which has been reported to increase the risk of cardio- and cerebrovascular events in comparison with non-smokers not exposed to smoke [28-30].

\section{Conclusion}

The present study showed that, at least in young and middle-aged smokers ( $\leq 59$ years old), physical activity did not demonstrate a beneficial effect on the maximum IMT of the carotid artery. It is possible that for current smokers, the favorable effect of physical activity on the prevention of carotid atherosclerosis will be offset by detrimental effect of smoking.

\section{Disclosure}

We have no conflicts to disclose.

\section{Grant Support}

This research was supported by internal funds only.

\section{References}

1. Sacco RL, Gan R, Boden-Albala B, Lin IF, Kargman DE, Hauser WA, Shea S, et al. Leisure-time physical activity and ischemic stroke risk: the Northern Manhattan Stroke Study. Stroke. 1998;29(2):380-387.

2. Elbaz A, Ripert M, Tavernier B, Fevrier B, Zureik M, Gariepy J, Alperovitch A, et al. Common carotid artery intima-media thickness, carotid plaques, and walking speed. Stroke. 2005;36(10):2198-2202.

3. Kadoglou NP, Iliadis F, Liapis CD. Exercise and carotid atherosclerosis. Eur J Vasc Endovasc Surg. 2008;35(3):264-272.

4. Sato S, Makita S, Uchida R, Ishihara S, Majima M. Physical activity and progression of carotid intima-media thickness in patients with coronary heart disease. $\mathrm{J}$ Cardiol. 2008;51(3):157-162.

5. You R, McNeil JJ, O’Malley HM, Davis SM, Donnan GA. Risk factors for lacunar infarction syndromes. Neurology. 1995;45(8):1483-1487.

6. Tell GS, Howard G, McKinney WM, Toole JF. Cigarette smoking cessation and extracranial carotid atherosclerosis. JAMA. 1989;261(8):1178-1180.

7. O'Leary DH, Polak JF, Kronmal RA, Manolio TA, Burke GL, Wolfson SK, Jr. Carotid-artery intima and media thickness as a risk factor for myocardial infarction and stroke in older adults. Cardiovascular Health Study Collaborative Research Group. N Engl J Med. 1999;340(1):14-22.

8. Evenson KR, Rosamond WD, Cai J, Toole JF, Hutchinson RG, Shahar E, Folsom AR. Physical activity and ischemic stroke risk. The atherosclerosis risk in communities study. Stroke. 1999;30(7):1333-1339.

9. Gillum RF, Mussolino ME, Ingram DD. Physical activity and stroke incidence in women and men. The NHANES I Epidemiologic Follow-up Study. Am J Epidemiol. 1996;143(9):860-869.

10. $\mathrm{Hu} \mathrm{G}$, Sarti C, Jousilahti P, Silventoinen K, Barengo NC, Tuomilehto J. Leisure time, occupational, and commuting physical activity and the risk of stroke. Stroke. 2005;36(9):1994-1999.

11. Lee CD, Folsom AR, Blair SN. Physical activity and stroke risk: a meta-analysis. Stroke. 2003;34(10):24752481.

12. Wendel-Vos GC, Schuit AJ, Feskens EJ, Boshuizen HC, Verschuren WM, Saris WH, Kromhout D. Physical activity and stroke. A meta-analysis of observational data. Int J Epidemiol. 2004;33(4):787-798.

13. Endres M, Gertz K, Lindauer U, Katchanov J, Schultze J, Schrock H, Nickenig G, et al. Mechanisms of stroke protection by physical activity. Ann Neurol. 2003;54(5):582-590.

14. Love BB, Biller J, Jones MP, Adams HP, Jr., Bruno A. Cigarette smoking. A risk factor for cerebral infarction in young adults. Arch Neurol. 1990;47(6):693-698.

15. Robbins AS, Manson JE, Lee IM, Satterfield S, Hennekens CH. Cigarette smoking and stroke in a cohort of U.S. male physicians. Ann Intern Med. 1994;120(6):458462.

16. Bhat VM, Cole JW, Sorkin JD, Wozniak MA, Malarcher AM, Giles WH, Stern BJ, et al. Dose-response relationship between cigarette smoking and risk of ischemic stroke in young women. Stroke. 2008;39(9):2439-2443.

17. Mast H, Thompson JL, Lin IF, Hofmeister C, Hartmann A, Marx P, Mohr JP, et al. Cigarette smoking as a determinant of high-grade carotid artery stenosis in Hispanic, black, and white patients with stroke or transient ischemic attack. Stroke. 1998;29(5):908-912.

18. Nakashima A, Yorioka N, Asakimori Y, Ito T, Masaki T, Shigemoto K, Harada S. Different risk factors for the maximum and the mean carotid intima-media thickness in hemodialysis patients. Intern Med. 2003;42(11):10951099.

19. Adachi H, Hirai Y, Fujiura Y, Matsuoka H, Satoh A, Imaizumi T. Plasma homocysteine levels and atherosclerosis in Japan: epidemiological study by use of carotid ultrasonography. Stroke. 2002;33(9):2177-2181.

20. Bots ML, Evans GW, Riley WA, Grobbee DE. Carotid intima-media thickness measurements in intervention 
studies. Stroke 2004; 34:2985-2994.

21. Wolf PA, D'Agostino RB, Kannel WB, Bonita R, Belanger AJ. Cigarette smoking as a risk factor for stroke. The Framingham Study. JAMA. 1988;259(7):10251029.

22. Kawachi I, Colditz GA, Stampfer MJ, Willett WC, Manson JE, Rosner B, Speizer FE, et al. Smoking cessation and decreased risk of stroke in women. JAMA. 1993;269(2):232-236.

23. Ainsworth BE, Haskell WL, Leon AS, Jacobs DR, Jr., Montoye HJ, Sallis JF, Paffenbarger RS, Jr. Compendium of physical activities: classification of energy costs of human physical activities. Med Sci Sports Exerc. 1993;25(1):71-80.

24. Craig CL, Marshall AL, Sjostrom M, Bauman AE, Booth ML, Ainsworth BE, Pratt M, et al. International physical activity questionnaire: 12-country reliability and validity. Med Sci Sports Exerc. 2003;35(8):1381-1395.

25. Riccioni G. Statins and carotid intima-media thickness reduction: an up-to-date review. Curr Med Chem.
2009;16(14):1799-1805.

26. Riccioni G. The effect of antihypertensive drugs on carotid intima media thickness: an up-to-date review. Curr Med Chem. 2009;16(8):988-996.

27. Katakami N, Kim YS, Kawamori R, Yamasaki Y. The phosphodiesterase inhibitor cilostazol induces regression of carotid atherosclerosis in subjects with type 2 diabetes mellitus: principal results of the Diabetic Atherosclerosis Prevention by Cilostazol (DAPC) study: a randomized trial. Circulation. 2010;121(23):2584-2591.

28. Zhang X, Shu XO, Yang G, Li HL, Xiang YB, Gao YT, Li Q, et al. Association of passive smoking by husbands with prevalence of stroke among Chinese women nonsmokers. Am J Epidemiol. 2005;161(3):213-218.

29. Bolego C, Poli A, Paoletti R. Smoking and gender. Cardiovasc Res. 2002;53(3):568-576.

30. He J, Vupputuri S, Allen K, Prerost MR, Hughes J, Whelton PK. Passive smoking and the risk of coronary heart disease--a meta-analysis of epidemiologic studies. N Engl J Med. 1999;340(12):920-926. 